UTAP-580, RESCEU-72/07

\title{
Reheating a multi-throat universe by brane motion
}

\author{
Shinji Mukohyama \\ Department of Physics and Research Center for the Early Universe, \\ The University of Tokyo, Tokyo 113-0033, Japan \\ Institute for the Physics and Mathematics of the Universe, University of Tokyo, \\ Chiba 277-8568, Japan
}

\begin{abstract}
We propose a mechanism of reheating after inflation in multi-throat scenarios of warped extra dimensions. Validity of an effective field theory on the standard model (SM) brane requires that the position of the SM brane during inflation be different from the position after inflation. The latter is supposed to be near the tip of the SM throat but the former is not. After inflation, when the Hubble expansion rate becomes sufficiently low, the SM brane starts moving towards the tip and eventually oscillates. The SM fields are excited by the brane motion and the universe is reheated. Since interaction between the brane position modulus and the SM fields is suppressed only by the local string scale, the modulus effectively decays into the SM fields.
\end{abstract}




\section{Introduction}

Our universe appears to have many mass scales, including the Planck scale, the electroweak scale, the scale of inflation, the scale of cosmic expansion today, etc. One of the most outstanding problems in theoretical physics is, hence, to explain how hierarchies among those scales emerge in a natural way.

The 5-dimensional braneworld scenario proposed by Randall and Sundrum generates the large hierarchy between the Planck scale and the electroweak scale by a warped 5th dimension [1]. Although their original scenario does not recover the ordinary 4-dimensional Einstein gravity at low energy on the brane confining the standard model (SM) of particle physics, this flaw was fixed by inclusion of a moduli stabilization mechanism [2].

Moduli stabilization is important not only for the Randall-Sundrum scenario but also for any models with extra dimensions. Unless all moduli are stabilized, the ordinary 4-dimensional Einstein gravity is not recovered and models with extra dimensions cannot be compatible with the universe we observe. (One interesting exception is the localized gravity [3].)

In type IIB string theory, all complex structure moduli are stabilized by turning on various fluxes. The geometry of extra dimensions are inevitably warped by those fluxes and, thus, this setup called warped flux compactification may be considered as a realization of the Randall-Sundrum scenario in string theory. A warped region of extra dimensions is called a warped throat and is supposed to have a regular tip corresponding to a local minimum of the warp factor. The 10-dimensional metric in the warped throat is represented as

$$
d s_{10}^{2}=h^{2} d s_{4}^{2}+h^{-2} d s_{6}^{2},
$$

where $d s_{4}^{2}=\eta_{\mu \nu} d x^{\mu} d x^{\nu}(\mu, \nu=0, \cdots, 3)$ is the 4-dimensional Minkowski metric, $d s_{6}^{2}$ is a Calabi-Yau metric on a 6-dimensional compact manifold and the warp factor $h$ depends only on the internal Calabi-Yau direction. In the construction by Giddings, Kachru and Polchinski (GKP) [4], the warp factor at the tip of a throat is given by

$$
h_{t i p} \simeq \exp \left(-\frac{2 \pi K}{3 g_{s} M}\right),
$$

where $M$ and $K$ are numbers of R-R and NS-NS fluxes, respectively, $g_{s}$ is the string coupling, and the warp factor is normalized to order unity in the unwarped, bulk Calabi-Yau region. As in the Randall-Sundrum scenario, this exponential warp factor generates a large hierarchy between the mass scale at the tip and that in the bulk 
Calabi-Yau region. For example, if the exponent in the right hand side of (1.2) is $\simeq-37$ then the local string scale at the tip is as low as the electroweak scale when the string scale in the bulk region is Planckian. The Einstein gravity is recovered at low energy if all other moduli, called Kähler moduli, are stabilized by non-perturbative effects such as D-instantons and/or gaugino condensation á la Kachru, Kallosh, Linde and Trivedi [5].

While those scenarios can address the hierarchy between the Planck scale and the electroweak scale, in our universe there are more than one hierarchies. In this respect it is tempting to consider more than one throats attached to a bulk CalabiYau manifold. Each throat has a tip corresponding to a local minimum of the warp factor and, thus, a characteristic mass scale, i.e. the local string scale. For example, a long throat may accommodate intersecting branes confining the SM fields and another throat with an intermediate length may accommodate a brane-antibrane pair to drive inflation [6]. In this way, the Planck scale in the bulk Calabi-Yau region, the electroweak scale at the tip of the SM throat and the scale of inflation at the tip of the inflaton throat can coexist in the geometrical setup. On the other hand, if both the SM and the inflaton are in a common throat then one might expect that supersymmetry might help accommodate both scales. However, this turns out to be rather difficult since the gravitino tends to be heavier than the Hubble scale during inflation [7, which is set to $H_{\text {inf }} \sim 10^{-5} \times \sqrt{\epsilon} M_{P l}$ by the amplitude of temperature fluctuations. Therefore, it seems plausible to consider more than one throats: one throat for the inflaton and another for the SM.

The purpose of this paper is to propose a new mechanism to reheat the SM fields after inflation in the multi-throat scenario. The rest of this paper is organized as follows. In Sec. 2 we point out a couple of problems with multi-throat inflation models. Motivated by those problems, in Sec. 3 we propose a reheating scenario. Sec. 4 is devoted to a summary of this paper.

\section{Problems with multi-throat inflation model}

In this section we point out a couple of problems with multi-throat inflation models.

\subsection{Reheating}

Once we adopt a multi-throat scenario, a natural question arises: "how to reheat the SM fields after inflation?" One of the reasons why this is not trivial is that the inflaton and the SM fields are living in different throats. In this subsection we shall 
review a known mechanism in the literature [8, 9, 10] and point out a problem. (See also [11].)

The end of a brane-antibrane inflation is annihilation of the brane-antibrane pair. When the inter-brane distance becomes as short as the local string length, a state of strings connecting the brane and the antibrane becomes tachyonic and it develops a non-vanishing expectation value. This "rolling" tachyon is similar to the waterfall direction in a usual field-theoretic hybrid inflation. The hight of the tachyon potential coincides with the sum of brane tension and antibrane tension and, according to Sen's conjecture [14], condensation of the tachyon towards a potential minimum is an effective description of the brane-antibrane annihilation.

For a non-vanishing string coupling, the "rolling" tachyon couples to closed strings. Highly excited closed strings are produced during the tachyon condensation and the energy stored in the brane-antibrane pair is rapidly dissipated within the local string time scale. Those excited closed string modes easily cascade down to massless closed string modes, i.e. 10-dimensional gravitons, again within the local string time scale. This cascade decay is mainly into Kaluza-Klein (KK) modes rather than 4-dimensional gravitons since the 4-dimensional gravitons are localized in the bulk Calabi-Yau region and KK modes are localized in the throat region. Indeed, since the brane-antibrane annihilation takes place deep inside the warped throat, it is rather natural to expect that the branching ratio of the decay into the 4-dimensional gravitons is exponentially suppressed compared with that into KK modes. Therefore, the inflaton throat will be filled with KK gravitons localized in the throat within the local string time scale after the emergence of the tachyon.

In the multi-throat scenario, the KK gravitons localized in the inflaton throat cannot be the eigen state of the Hamiltonian even in the quadratic order. This situation is similar to solving Schrödinger equation with a double well potential. The lowest eigen state in this quantum mechanical problem is symmetric and the second lowest eigen state is antisymmetric so that a state localized near one of the two local minima is a linear combination of the symmetric and antisymmetric states. Since these two eigen states have different energies, the linear combination is not an eigen state of the Hamiltonian and, thus, evolves to different linear combinations of the eigen states as time proceeds. In other words, the peak of the wavefunction oscillates between two minima. The time scale of the oscillation is given by the inverse of the energy difference between the two eigen states. We expect a similar phenomenon for the KK modes localized in one of throats in multi-throat situation: the peak of the KK graviton wave functions should oscillate among throats [12, 13]. When a KK graviton wave function has a peak in the SM throat during the oscillation, the KK 
graviton can interact with and decay into the SM fields.

Successful reheating is possible only if the decay into the SM fields dominates over the decay into the 4-dimensional gravitons. The former is suppressed by the relatively long time scale of the oscillation of the KK graviton wave functions. On the other hand, the latter, which can happen at any stages of reheating, is suppressed by the warping of the throats. The result of refs. [8, 9, 10] indicates that the decay into the SM field indeed dominates over that into the 4-dimensional gravitons for a range of parameters.

In multi-throat scenarios with the inflaton throat and the SM throat, it is natural to expect that there are more than two throats and that there are branes in some of them. If there are other branes then light fields on them are also excited by the above chain of processes. For example, if there is a throat with a stack of D-branes at the tip then gauge fields on it will be excited. Moreover, if the initial numbers of branes and antibranes in the inflaton throat are not the same then there will be leftover branes or antibranes and gauge fields on them will be excited by more direct processes. For the success of big-bang nucleo synthesis, we have to make it sure that more than $90 \%$ of energies distributed over many branes is going into the SM brane.

\subsection{Breakdown of the effective field theory}

A more severe problem is breakdown of the low-energy effective field theory (EFT) on the SM brane during inflation.

The local string scale in warped compactification depends on the physical position in extra dimensions. In multi-throat scenarios, since the warp factor varies from throat to throat, each throat has each local string scale at the tip. Since one of the main motivations for considering multi-throat scenarios is to accommodate more than one mass hierarchies in a natural way, it is plausible to suppose that the scale of inflation and the electroweak scale are essentially the local string scales at the tip of the inflaton throat and the SM throat, respectively. In this case, since the Hubble scale during inflation is much higher than the electroweak scale, the local string scale at the tip of the SM throat is expected to be much lower than the Hubble scale during inflation.

The above argument is based on the picture that the 4-dimensional Planck scale is fixed while the local string scale changes as a brane of interest moves in extra dimensions. In other words, this picture sets the scale of reference by the universal 4-dimensional metric $d s_{4}^{2}$ in (1.1). Alternatively, we could use the metric $h^{2} d s_{4}^{2}$ to set the scale of reference. In this case the string scale would be universal everywhere since this metric corresponds to the induced string metric on the brane. On the 
other hand, in this picture the 4-dimensional Planck scale would change as a brane of interest moves in extra dimensions. Nonetheless, the ratio of the string scale to the 4-dimensional Planck scale at the position of a brane in this picture should be the same as that in the previous picture. Actually, as far as dimensionless physical quantities such as ratios among mass scales are concerned, the two pictures give the same answer as they should. In this paper we adopt the former picture in which the 4-dimensional Planck scale is universal. Note that a mass scale is dimensionful and, thus, it has physical meaning only when it is compared with another mass scale. For example, the ratio of the local string scale at the position of a brane to the 4-dimensional Planck scale has physical meaning irrespective of a scale of reference.

Coming back to the issue of breakdown of the EFT, if the SM brane is at the tip of the SM throat then stringy effects become dominant on the SM brane during inflation since the Hubble scale exceeds the local string scale. This leads to breakdown of the EFT on the SM brane. In this case we expect violent physical processes such as production of black holes, strings, topological defects and so on. At the very least, cosmological density perturbations should significantly deviate from the Gaussian distribution due to nonlinear natures of the strongly coupled physics and will most likely contradict with current observation.

\section{A scenario}

The aim of this section is to propose a reheating scenario without breakdown of the EFT on the SM brane.

The position of the SM brane in extra dimensions is a modulus field. At low energy this modulus field is supposed to be stabilized near the tip of the SM throat. On the other hand, during inflation, a moduli potential usually obtain $H$-dependent corrections, where $H$ is the Hubble scale during inflation [15]. For example, a modulus field can obtain a $H$-dependent mass term and the origin of the $H$-dependent mass term does not have to be at the tip of a throat 1 . Indeed, since a multi-throat configuration is somehow similar to a double-well potential as discussed in subsection 2.1, it seems natural to expect that the origin of the $H$-dependent mass term is in or near the bulk region. Thus, it is plausible that the $H$-dependent corrections to the moduli potential shift the minimum of the potential for the position of the SM

\footnotetext{
${ }^{1}$ The derivation of the $H$-dependent mass term via supergravity does not specify the origin of the $H$-dependent mass term: the dependence of the Kähler potential on the brane position is determined so that it recovers the correct kinetic term for the brane position [16], which does not depend on the origin.
} 
brane. The length of the SM throat is also a modulus and is expected to be shifted by $H$-dependent corrections to its potential [17]. In the following we suppose that the modulus corresponding to the brane position is lighter than the modulus corresponding to the length of the throat. There may be branes other than the SM brane and the brane-antibrane pair, and their positions are also moduli. They also start moving when the Hubble scale becomes comparable with their mass, and oscillate around the potential minima. Considering the fact that there is no hierarchal mass scales between the electroweak scale and today's Hubble scale, we assume that there is no moduli with masses in the range between these two scales. In this case, the SM brane will be the one that started oscillating last. For this reason, in the following we shall consider the motion of the SM brane only.

For an EFT to be valid on the SM brane, the local string scale at its position during inflation must be higher than the Hubble scale and, thus, much higher than the local string scale at the tip of the SM throat. This means that the validity of the EFT requires that the minimum of the potential for the SM brane position during inflation should be significantly different from that for $H=0$. This is possible, for example, if the origin of the $H$-dependent mass term for the brane position is in or near the bulk Calabi-Yau region. In this case the SM brane will start moving towards the tip of the SM throat when the Hubble expansion rate becomes sufficiently low after inflation. Before this epoch, the energy stored in the brane-antibrane pair is distributed to many light fields on various branes by the chain of processes explained in subsection 2.1 and is diluted. Eventually, the SM brane will oscillate around the tip of the throat [18] and fields on the SM brane will be excited due to interactions with the brane position modulus. This kind of interaction is in general suppressed only by the local string scale (but not by the Planck scale) and, thus, the modulus effectively decays into the SM fields.

Of course, after the SM brane settles down near the tip of the throat, the modulus field corresponding to the position of the SM brane is massive and does not affect physics at sufficiently low energies.

\subsection{A toy model: $U(1)$ on wrapped D5}

In type IIB superstring theory the SM may be realized on intersecting $D$-branes. In this subsection, to see that the motion of the SM brane excites fields on it, we consider a simpler toy model: a wrapped $D 5$-brane and a $U(1)$ field on it. We shall see that the $U(1)$ field is indeed excited by the homogeneous motion of the D5-brane.

The action for a probe D5-brane is given as the sum of Dirac-Born-Infeld and 
Chern-Simon terms

$$
S_{D 5}=-T_{5} \int d^{6} \xi e^{-\Phi} \sqrt{-\operatorname{det}\left(G_{A B}-B_{A B}-2 \pi \alpha^{\prime} F_{A B}\right)}+T_{5} \int d^{6} \xi B \wedge C_{4},
$$

where $\xi^{A}(A=0, \cdots, 5)$ are intrinsic coordinates on the brane, $T_{5}$ is the brane tension, $\Phi$ is the dilaton, $G_{A B}$ is the induced metric on the brane, $B_{A B}$ and $C_{4}$ are pullbacks of the NS-NS antisymmetric field and the R-R 4-form potential on the brane worldvolume, and $F_{A B}$ is the field strength of the $U(1)$ gauge field on the brane. For simplicity we assume that the dilaton is stabilized to zero as in the Klebanov-Strassler throat [19] with the GKP construction, and consider the warped geometry of the form (1.1) with

$$
d s_{6}^{2}=d r^{2}+d s_{5}^{2}
$$

where $d s_{5}^{2}$ is an $r$-dependent 5-dimensional metric.

We consider radial motion of a D5-brane wrapped over a 2-cycle in the warped geometry and shall see that the homogeneous motion of the brane excites the 4dimensional part of the $U(1)$ field. We adopt a gauge in which first four brane coordinates $\xi^{\mu}(\mu=0, \cdots, 3)$ coincide with $x^{\mu}$ and the other brane coordinates $\xi^{p}$ $(p=4,5)$ coincide with coordinates on the 2-cycle, respectively, and suppose that $r$ depends only on $\xi^{\mu}$. In this case,

$$
G_{A B} d \xi^{A} d \xi^{B}=h^{2}\left(\eta_{\mu \nu}+h^{-4} \partial_{\mu} r \partial_{\nu} r\right) d \xi^{\mu} d \xi^{\nu}+h^{-2} \sigma_{p q} d \xi^{p} d \xi^{q}
$$

where $\sigma_{p q} d \xi^{p} d \xi^{q}$ is the pullback of the 5 -dimensional metric $d s_{5}^{2}$ on the 2-cycle. Thus, we obtain

$$
-\operatorname{det}\left(G_{A B}-B_{A B}\right)=h^{4}\left(1+h^{-4} \eta^{\mu \nu} \partial_{\mu} r \partial_{\nu} r\right)\left(1+B^{2}\right) \sigma
$$

where $\sigma \equiv \operatorname{det} \sigma_{p q}$ and $B$ is defined by

$$
B_{A B} d \xi^{A} d \xi^{B}=h^{-2} B \sqrt{\sigma} \epsilon_{p q} d \xi^{p} d \xi^{q}
$$

Hereafter, we assume that $h$ and $B$ are functions of $r$ only and consider a homogeneous brane configuration $r=r\left(\xi^{0}\right)$. Note that $B_{\mu \nu}$ and $B_{\mu p}=-B_{p \mu}$ have been set to zero in order to avoid breaking the 4-dimensional local Lorentz invariance.

As for $F_{A B}$, for simplicity we set $F_{\mu p}=-F_{p \mu}=0$ and $F_{p q}=0$. Up to the order $O\left(F^{2}\right)$, this is a consistent truncation since these components decouple from $F_{\mu \nu}$ in the action up to this order. We also assume that $F_{\mu \nu}$ depends on the 4dimensional coordinates $x^{\mu}$ only, i.e. we drop massive Kaluza-Klein modes. This is also a consistent truncation up to the order $O\left(F^{2}\right)$. Up to the second order in $F_{\mu \nu}$, 
we obtain

$$
\frac{\sqrt{-\operatorname{det}\left(G_{A B}-B_{A B}-2 \pi \alpha^{\prime} F_{A B}\right)}}{\sqrt{-\operatorname{det}\left(G_{A B}-B_{A B}\right)}}=1-\frac{\left(2 \pi \alpha^{\prime}\right)^{2}}{2 h^{4}}\left(\frac{\delta^{i j} F_{0 i} F_{0 j}}{1-\left(\partial_{0} r\right)^{2} / h^{4}}-\frac{1}{2} \delta^{i j} \delta^{k l} F_{i k} F_{j l}\right),
$$

where $i, j=1,2,3$. Thus, after integration over the 2 -cycle we obtain

$$
S_{D 5}=\int d \xi^{4}\left[-\frac{T(\phi)}{\tilde{\gamma}}+T(\phi)-V(\phi)+\frac{\delta^{i j}}{2 g^{2}(\phi)}\left(\tilde{\gamma} F_{0 i} F_{0 j}-\frac{1}{2 \tilde{\gamma}} \delta^{k l} F_{i k} F_{j l}\right)\right],
$$

where

$$
\begin{aligned}
T(\phi) & \equiv T_{5} v_{2} h^{2} \sqrt{1+B^{2}} \\
\frac{1}{g^{2}(\phi)} & \equiv\left(2 \pi \alpha^{\prime}\right)^{2} T / h^{4} \\
\tilde{\gamma} & \equiv \frac{1}{\sqrt{1-\left(\partial_{0} \phi\right)^{2} / T(\phi)}}
\end{aligned}
$$

and we have expressed the contribution from the Chern-Simon term as $T-V$. Here, $\phi$ is a field defined by

$$
d \phi=\left(T_{5} v_{2} h^{-2} \sqrt{1+B^{2}}\right)^{1 / 2} d r
$$

and $v_{2}$ is the (unwarped) volume of the 2-cycle

$$
v_{2} \equiv \int d \xi^{4} d \xi^{5} \sqrt{\sigma}
$$

which depends on $\phi$.

Now, assuming that the physical energy scale associated with the 4-dimensional universe is much lower than the energy scale of moduli stabilization, we promote the 4-dimensional effective action to the FRW background. By replacing $\eta_{\mu \nu} d \xi^{\mu} d \xi^{\nu}$ with $a^{2}\left(\xi^{0}\right) \eta_{\mu \nu} d \xi^{\mu} d \xi^{\nu}$ and repeating the above procedure, we obtain $S_{D 5}=S_{\phi}+S_{A}$, where

$$
\begin{aligned}
S_{\phi} & =\int d \xi^{4} a^{4}\left[-\frac{T(\phi)}{\gamma}+T(\phi)-V(\phi)\right], \\
S_{A} & =\int d \xi^{4} \frac{\delta^{i j}}{2 g^{2}(\phi)}\left(\gamma F_{0 i} F_{0 j}-\frac{1}{2 \gamma} \delta^{k l} F_{i k} F_{j l}\right),
\end{aligned}
$$

where

$$
\gamma \equiv \frac{1}{\sqrt{1-a^{-2}\left(\partial_{0} \phi\right)^{2} / T(\phi)}}
$$

Note that both $S_{\phi}$ and $S_{A}$ should in general receive corrections due to moduli stabilization, radiative corrections, curvature corrections and so on. However, the 
$U(1)$ gauge symmetry and the spatial rotational invariance protect the form of $S_{A}$. (The form of $\gamma$ and $g^{2}(\phi)$ may change.) What is important in the following analysis is the form of $S_{A}$ only.

Let us now see that the $U(1)$ field should be excited by the homogenous motion of the brane. This is similar to the decay of a moduli field into gauge field via the dependence of the gauge coupling on the moduli field. By setting $A_{0}=0$ and $\delta^{i j} \partial_{i} A_{j}=0$, we obtain

$$
S_{A}=\int d \xi^{4} \frac{\delta^{i j}}{2 g^{2}(\phi)}\left(\gamma \partial_{0} A_{i} \partial_{0} A_{j}-\frac{\delta^{k l}}{\gamma} \partial_{k} A_{i} \partial_{l} A_{j}\right)
$$

and the corresponding equation of motion is

$$
\gamma g^{2} \frac{d}{d \eta}\left(\frac{\gamma}{g^{2}} \frac{d A_{i}}{d \eta}\right)-\delta^{k l} \partial_{k} \partial_{l} A_{i}=0
$$

where $\eta=\xi^{0}$. During inflation, $\phi$ stays almost constant and, thus, $\gamma \simeq 1$ and $g^{2}$ is almost constant. Thus, $A_{i}$ is expanded as

$$
A_{i}=\int \frac{d^{3} \vec{k}}{\sqrt{(2 \pi)^{3}}} \sum_{I=1,2} e_{i}^{I}(\vec{k})\left[a_{I}(\vec{k}) \varphi(\vec{k}, \eta) e^{i \vec{k} \cdot \vec{\xi}}+a_{I}^{\dagger}(\vec{k}) \varphi^{*}(\vec{k}, \eta) e^{-i \vec{k} \cdot \vec{\xi}}\right]
$$

where $e_{i}^{I}(I=1,2)$ is the polarization vector satisfying

$$
e^{I} \cdot e^{J}=\delta^{I J}, \quad e^{I} \cdot \vec{k}=0,
$$

and $\varphi$ satisfies

$$
\varphi \simeq \frac{g_{\text {inf }}}{\sqrt{2|\vec{k}|}} e^{-i|\vec{k}| \eta}
$$

during inflation, and

$$
\gamma g^{2} \frac{d}{d \eta}\left(\frac{\gamma}{g^{2}} \frac{d \varphi}{d \eta}\right)+|\vec{k}|^{2} \varphi=0
$$

for all the time since then. Here, $g_{i n f}$ is the (almost constant) value of $g$ during inflation. Long after inflation, the modulus $\phi$ settles down to the value at the minimum of its potential and, thus, $\gamma \simeq 1$ and $g^{2}$ approaches a constant. Thus, $A_{i}$ is expanded as

$$
A_{i}=\int \frac{d^{3} \vec{k}}{\sqrt{(2 \pi)^{3}}} \sum_{I=1,2} e_{i}^{I}(\vec{k})\left[\tilde{a}_{I}(\vec{k}) \tilde{\varphi}(\vec{k}, \eta) e^{i \vec{k} \cdot \vec{\xi}}+\tilde{a}_{I}^{\dagger}(\vec{k}) \tilde{\varphi}^{*}(\vec{k}, \eta) e^{-i \vec{k} \cdot \vec{\xi}}\right]
$$

where $\tilde{\varphi}$ satisfies

$$
\tilde{\varphi} \simeq \frac{g_{0}}{\sqrt{2|\vec{k}|}} e^{-i|\vec{k}| \eta}
$$


long after inflation, and

$$
\gamma g^{2} \frac{d}{d \eta}\left(\frac{\gamma}{g^{2}} \frac{d \tilde{\varphi}}{d \eta}\right)+|\vec{k}|^{2} \tilde{\varphi}=0
$$

until then. Here, $g_{0}$ is the asymptotic value of $g$ at late time. Since $g^{2}$ is timedependent between the two asymptotic regimes, the two sets of mode functions $\varphi$ and $\tilde{\varphi}$ are not identical. (The time dependence of $\gamma$ can be absorbed by redefinition of the time variable.) Instead, they are related as

$$
\tilde{\varphi}(\vec{k}, \eta)=\alpha(\vec{k}) \varphi(\vec{k}, \eta)+\beta(\vec{k}) \varphi^{*}(\vec{k}, \eta)
$$

Hence, if we start with the initial vacuum defined by

$$
a_{I}(\vec{k})|i n\rangle=0 \quad \text { for }{ }^{\forall} I \text { and }{ }^{\forall} \vec{k}
$$

then the number of created particles at late time is

$$
\left\langle i n\left|\tilde{a}_{I}^{\dagger}(\vec{k}) \tilde{a}_{I}(\vec{k})\right| i n\right\rangle=|\beta(\vec{k})|^{2} \neq 0 .
$$

This shows that the motion of the wrapped $D 5$-brane excites the $U(1)$ field on it.

The above analysis explicitly shows that the neutral modulus field $\phi$ can directly decay into the $U(1)$ gauge field. Up to the order $O\left(F_{\mu \nu}^{2}\right)$, the decay is due to the coupling of the modulus field to the gauge field via the dependence of the gauge coupling on the modulus field. On the other hand, in higher order, there are many other decay channels although we probably have less theoretical controls. In this sense, what we have seen above is just one of many possible processes to excite the $U(1)$ field by the brane motion.

After all, oscillation of the SM brane is rather violent phenomenon: the brane loading all components of the SM, including ourselves, is moving! It is not at all unreasonable to expect that excitation of the SM fields due to the brane motion is efficient.

\subsection{Reheating temperature}

As we have seen in the previous subsection, the SM fields can be excited by motion of the SM brane. Processes include non-perturbative [20] as well as perturbative decays of the modulus field. In this subsection, we shall estimate the reheating temperature based on the decay rate in the perturbative regime (with small amplitude of the oscillation of $\phi$ ). Processes in the non-perturbative regime (with large amplitude of the oscillation of $\phi$ ) are certainly worthwhile investigating as a future work. 
In scenarios with warped extra dimensions, it is expected that interaction between the modulus corresponding to the brane position and fields on the brane is suppressed not by the Planck scale but by the local string scale. To see this let us suppose that at low energy, a D5-brane wrapped over a 2-cycle is stabilized somewhere, say at $r=r_{0}$, in a warped throat. We assume that the warp factor at $r=r_{0}$ is small enough to address the hierarchy problem. Because of the warped geometry, dimension-full quantities on the brane should scale as some powers of the warp factor. In particular, unless fine-tuned, we expect that

$$
\frac{d \ln g}{d \phi} \sim \frac{C\left(g_{s}\right)}{M_{\text {local }}}
$$

where $M_{\text {local }}$ is the local string scale defined by

$$
M_{\text {local }} \equiv \frac{h_{t i p}}{\sqrt{\alpha^{\prime}}}
$$

(Note that $\phi$ has been normalized so that its leading kinetic term is canonical.)

Thus, Lagrangian is expanded near the present value $\phi=\phi_{0}$ as

$$
-\frac{1}{4 g_{0}^{2}}\left[F^{\mu \nu} F_{\mu \nu}+\frac{O(1)}{M_{\text {local }}}\left(\phi-\phi_{0}\right) F^{\mu \nu} F_{\mu \nu}+\cdots\right] \text {. }
$$

Therefore, in this toy model the interaction between the modulus $\phi$ and the $U(1)$ gauge field is suppressed by the local string scale. Note that the warp factor $h$ at $r=r_{0}$ is assumed to be exponentially small (see (1.2) ) so that we have the hierarchy

$$
M_{\text {local }} \ll M_{P l},
$$

where $M_{P l}$ is the 4-dimensional Planck scale given by

$$
M_{P l}^{2} \simeq \frac{2 V_{6}}{(2 \pi)^{7} \alpha^{\prime 4} g_{s}^{2}}
$$

Here, $V_{6}$ is the volume of the bulk Calabi-Yau region.

Since the suppression of the moduli interaction is just by the local string scale, it is expected that the decay rate of $\phi$ into the SM fields in the perturbative regime is

$$
\Gamma \sim \frac{m_{\phi}^{3}}{M_{\text {local }}^{2}}
$$

where the local string scale $M_{\text {local }}$ is given by (3.26). Correspondingly, the reheating temperature is estimated as

$$
T_{\text {reh }} \sim \frac{\sqrt{M_{P l} m_{\phi}^{3}}}{M_{\text {local }}} .
$$

Since $M_{P l}$ is not in the denominator, it is easy to make the reheating temperature sufficiently high. 


\section{Summary}

More than one mass hierarchies can coexist in multi-throat scenarios of warped extra dimensions. A long throat can accommodate a brane confining the standard model (SM) of particle physics while another throat with an intermediate length can accommodate a brane-antibrane pair to drive inflation. In this paper, after pointing out a couple of problems with the multi-throat inflation scenario, we have proposed a new reheating mechanism in which motion of the SM brane excites fields on it. Since interaction is suppressed only by the local string scale, the modulus corresponding to the brane position effectively decays into the SM fields. We have considered a wrapped $D 5$-brane as a simple toy model of the SM brane and show that the brane motion indeed excites the $U(1)$ gauge field on it. The decay process that we have explicitly investigated is similar to the decay of a moduli field into a gauge field via moduli dependence of the gauge coupling, but in general there are many other decay channels.

In some sense, this is an illustration how the moduli problem reappears and is solved in brane world scenarios with warped extra dimensions. In the present case, interaction between the moduli field and the SM fields is suppressed not by the Planck scale but by the local string scale at the position of the SM brane. Since the local string scale is much lower than the Planck scale, the moduli effectively decays and reheats the universe. Positions of other branes are also moduli but they decay before the SM brane, provided that they are heavier than the electroweak scale as discussed in the beginning of Sec. 3 .

In order to estimate reheating temperature, it is important to consider more realistic models of the SM brane such as intersecting $D$-branes and investigate how the brane motion and fields on it interact with each other. It is also worthwhile understanding how this mechanism of reheating can be understood in terms of scalar-tensor theory.

\section{Acknowledgements}

The author would like to thank J. Yokoyama for helpful discussions and L. Kofman for useful comments. This work was in part supported by MEXT through a Grant-inAid for Young Scientists (B) No. 17740134 and by JSPS through a Grant-in-Aid for Creative Scientific Research No. 19GS0219 and through a Grant-in-Aid for Scientific Research (B) No. 19340054. 


\section{References}

[1] L. Randall and R. Sundrum, "A large mass hierarchy from a small extra dimension," Phys. Rev. Lett. 83, 3370 (1999) arXiv:hep-ph/9905221.

[2] W. D. Goldberger and M. B. Wise, "Modulus stabilization with bulk fields," Phys. Rev. Lett. 83, 4922 (1999) arXiv:hep-ph/9907447].

[3] L. Randall and R. Sundrum, "An alternative to compactification," Phys. Rev. Lett. 83, 4690 (1999) arXiv:hep-th/9906064.

[4] S. B. Giddings, S. Kachru and J. Polchinski, "Hierarchies from fluxes in string compactifications," Phys. Rev. D 66, 106006 (2002) [arXiv:hep-th/0105097].

[5] S. Kachru, R. Kallosh, A. Linde and S. P. Trivedi, "De Sitter vacua in string theory," Phys. Rev. D 68, 046005 (2003) [arXiv:hep-th/0301240].

[6] S. Kachru, R. Kallosh, A. Linde, J. M. Maldacena, L. McAllister and

S. P. Trivedi, "Towards inflation in string theory," JCAP 0310, 013 (2003) arXiv:hep-th/0308055.

[7] R. Kallosh and A. Linde, "Landscape, the scale of SUSY breaking, and inflation," JHEP 0412, 004 (2004) arXiv:hep-th/0411011.

[8] N. Barnaby, C. P. Burgess and J. M. Cline, "Warped reheating in brane-antibrane inflation," JCAP 0504, 007 (2005) arXiv:hep-th/0412040.

[9] L. Kofman and P. Yi, "Reheating the universe after string theory inflation," Phys. Rev. D 72, 106001 (2005) arXiv:hep-th/0507257].

[10] D. Chialva, G. Shiu and B. Underwood, "Warped reheating in multi-throat brane inflation," JHEP 0601, 014 (2006) arXiv:hep-th/0508229].

[11] J. H. Brodie and D. A. Easson, "Brane inflation and reheating," JCAP 0312, 004 (2003) arXiv:hep-th/0301138.

[12] S. Dimopoulos, S. Kachru, N. Kaloper, A. E. Lawrence and E. Silverstein, "Small numbers from tunneling between brane throats," Phys. Rev. D 64, 121702 (2001) arXiv:hep-th/0104239.

[13] S. Dimopoulos, S. Kachru, N. Kaloper, A. E. Lawrence and E. Silverstein, "Generating small numbers by tunneling in multi-throat compactifications," Int. J. Mod. Phys. A 19, 2657 (2004) arXiv:hep-th/0106128. 
[14] A. Sen, "Rolling tachyon," JHEP 0204, 048 (2002) arXiv:hep-th/0203211.

[15] M. Dine, L. Randall and S. D. Thomas, "Baryogenesis From Flat Directions Of The Supersymmetric Standard Model," Nucl. Phys. B 458, 291 (1996) arXiv:hep-ph/9507453.

[16] O. DeWolfe and S. B. Giddings, "Scales and hierarchies in warped compactifications and brane worlds," Phys. Rev. D 67, 066008 (2003) arXiv:hep-th/0208123.

[17] A. R. Frey, A. Mazumdar and R. Myers, "Stringy effects during inflation and reheating," Phys. Rev. D 73, 026003 (2006) arXiv:hep-th/0508139.

[18] S. Mukohyama, "anti-D-brane as dark matter in warped string compactification," Phys. Rev. D 72, 061901 (2005) arXiv:hep-th/0505042.

[19] I. R. Klebanov and M. J. Strassler, "Supergravity and a confining gauge theory: Duality cascades and chiSB-resolution of naked singularities," JHEP 0008, 052 (2000) arXiv:hep-th/0007191.

[20] L. Kofman, A. D. Linde and A. A. Starobinsky, "Towards the theory of reheating after inflation," Phys. Rev. D 56, 3258 (1997) arXiv:hep-ph/9704452. 